\title{
Le contrôle de qualité externe en Biologie de la Reproduction de 1992 à 1996
}

\author{
Bourguignat A. *, Clavert A.**, Férard G.*
}

*Université Louis Pasteur, Faculté de Pharmacie, Service de Biochimie Appliquée, B.P. 24, 67401 Illkirch Cedex ; **Université Louis Pasteur, Faculté de Médecine, Laboratoire de Biologie de la Reproduction, Hôpitaux Universitaires de Strasbourg, 1 place de l'Hôpital, 67000 Strasbourg

\section{RESUME}

L'utilisation des résultats de laboratoire dans l'aide à la décision nécessite d'avoir des données fiables, donc une assurance dans la qualité des analyses effectuées. Nous avons évalué celle-ci à l'aide d'un Contrôle de Qualité Externe (CQE) de 1992 à 1996, chaque laboratoire analysant les mêmes spécimens.

Trente sept participants ont suivi cette procédure en évaluant les deux critères les plus courants du spermocytogramme: la proportion de spermatozoïdes normaux et le degré de tératospermie (à l'aide de l'Indice d'Anomalies Multiples, IAM). L'interprétation des résultats en termes de précision et d'exactitude a été effectuée comme pour les CQE de Biochimie, montrant par la même occasion la transférabilité des méthodes d'évaluation de la qualité des résultats dans un domaine où l'observation remplace la mesure.

En quatre ans, la dispersion des résultats concernant l'évaluation de la proportion des spermatozoïdes normaux est toujours aussi mauvaise (de 30 à $40 \%$ ), alors qu'une nette amélioration de l'évaluation de l'IAM a été notée (passant de 25 à $15 \%$ pendant la même période).

Après quatre années de participation à un CQE, l'approche d'une telle démarche est justifiée et doit être améliorée ; d'autres paramètres utilisés dans l'exploration de l'infertilité masculine devraient aussi être étudiés.

Mots clés : Contrôle de Qualité-Spermocytogramme

\section{INTRODUCTION}

La Biologie de la Reproduction est une discipline ancienne qui, pour des raisons sociologiques, n'a trouvé que tardivement des applications en médecine. Son développement a par contre été explosif depuis les années 1970 et continue à être un domaine en pleine évolution. Les innovations techniques s'y succèdent toujours à des rythmes accélérés et ont permis des progrès inespérés ; mais il s'est avéré très rapidement que l'interprétation des résultats des analyses était difficile car souvent basée sur des paramètres mal définis donc difficilement comparables d'une équipe à l'autre, donnant lieu à des séries statistiques d'interprétation délicate et à des conclusions quelquefois divergentes. D'où la nécessité d'une réflexion sur l'Assurance de Qualité en Biologie de la Reproduction. Une des premières démarches dans ce domaine consiste donc à mieux définir ce que l'on veut analyser et à harmoniser réalisation et interprétation des résultats pour augmenter l'efficacité diagnostique des examens de laboratoire et réduire leur répétition souvent délicate pour les patient(e)s. 
En effet, le pouvoir informant (diagnostique ou pronostique) d'une analyse vis-à-vis d'une pathologie définie, est étroitement lié à la partie analytique qui peut être améliorée notamment par la mise en place d'un contrôle de qualité. Les efforts effectués dans le domaine de la Biochimie Clinique depuis les années 1975 ont permis cette progression et notre démarche aujourd'hui est d'essayer d'utiliser la même approche dans le domaine de la Biologie de la Reproduction.

Rappelons brièvement qu'un bon marqueur biochimique se doit de pouvoir présenter des variations fortement associées à la présence d'une pathologie (notion de sensibilité), au contraire, ses non-variations seront assosciées à l'absence de la pathologie (notion de spécificité), l'ensemble sensibilité et spécificité correspondant à l'efficacité clinique. Pour des raisons évidentes, plus les variations analytiques sont faibles, plus le marqueur sera efficace dans l'aide au diagnostic ou au pronostic et inversement. Les critères d'évaluation les plus utilisés sont la précision (qualité de l'accord entre une série de mesures sur un même échantillon, dans les mêmes conditions opératoires) et l'exactitude (qualité de l'accord entre le résultat d'une mesure et la valeur vraie de ce constituant en dehors des erreurs de précision). A titre d'exemple, dans le cadre d'une association régionale de Contrôle de Qualité (Club Région Est de Contrôle de Qualité, CRECQ *), on a pu observer que les variations interlaboratoires du cholestérol total mesurées dans la région Grand Est de la France sont passées de 18 à $4 \%$ en 5 ans à la suite de la mise en place d'un contrôle de qualité de ce paramètre (Figure 1).

Le spermocytogramme étant l'examen de première intention en spermiologie, il est normal que nous nous y soyons intéressés en premier lieu et nous rapportons ici les résultats de l'application en spermiologie des méthodologies employées en Biochimie Clinique pour évaluer la qualité des résultats dans le cadre d'un Contrôle de Qualité Externe (CQE).

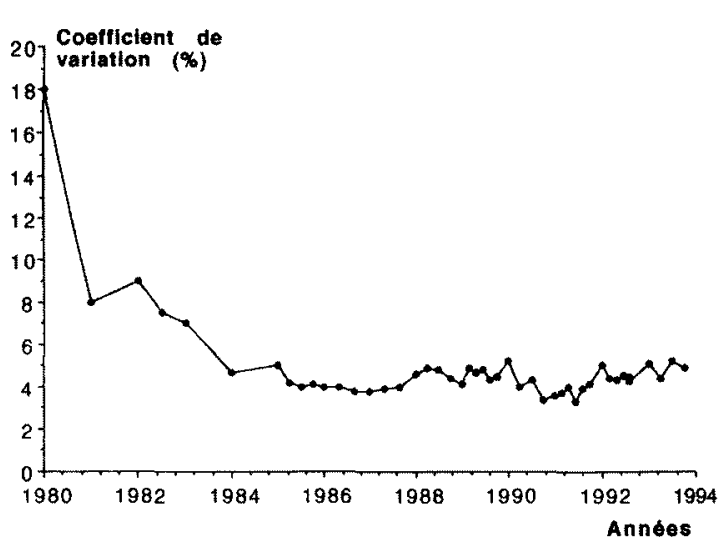

Figure 1 : Evolution de la dispersion interlaboratoire des résultats du dosage du cholestérol total dans la région Nord-Est de la France de 1980 à 1994.

\section{MATERIEL ET METHODES}

La mise en place en 1992 d'un CQE a été basée sur la notion de volontariat de la part des laboratoires de Biologie. Après inscription, chacun d'entre eux est identifié par un code confidentiel inconnu des autres laboratoires. Les participants reçoivent deux lames préfixées provenant chacune d'un même éjaculat. La coloration est effectuée par le laboratoire selon sa technique habituelle. Dans un premier temps, nous nous sommes intéressés aux deux paramètres les plus importants de l'examen du spermocytogramme : la proportion de spermatozoïdes normaux et l'étude du degré de tératospermie par le calcul de l'Indice d'Anomalies Multiples (IAM), [1, 2]. Chaque laboratoire retourne ses résultats à l'organisme centralisateur sous son numéro confidentiel afin de préserver l'anonymat des réponses.

Le traitement statistique de l'ensemble des données est effectué en terme de moyenne, écart-type et coefficient de variation (CV). Ce dernier paramètre, exprimé en \% et calculé selon la formule $\mathrm{CV}=$ (écart-type/ moyenne) x 100 , permet d'apprécier la dispersion des valeurs autour de la moyenne. Toutefois, comme les résultats en Biologie de la Reproduction peuvent être très éloi- 
gnés les uns des autres, nous avons introduit le calcul de la médiane qui correspond à la valeur pour laquelle la moitié des résultats lui est inférieure et l'autre moitié supérieure.

Enfin, pour pouvoir apprécier précision et exactitude, nous avons utilisé le diagramme de Youden [3] construit de la manière suivante (Figure 2) : pour un paramètre donné, chaque laboratoire est représenté sur un graphique par un point correspondant aux deux valeurs rendues pour ce paramètre : abcisse pour le résultat de la lame $n^{\circ} 1$, ordonnée pour la lame $n^{\circ} 2$. La valeur cible (définie ci-dessous) est reportée ainsi que les valeurs limites d'acceptabilité (prenant en compte la variabilité analytique et l'état de l'art notamment). On obtient ainsi un rectangle (ou un cercle) définissant une zone d'acceptabilité des résultats avec ses bornes supérieures et inférieures pour les deux paramètres analysés, et d'autres zones mettant en évidence des écarts plus ou moins importants vis à vis de la valeur cible :
Droite de précision mais d'inexactitude

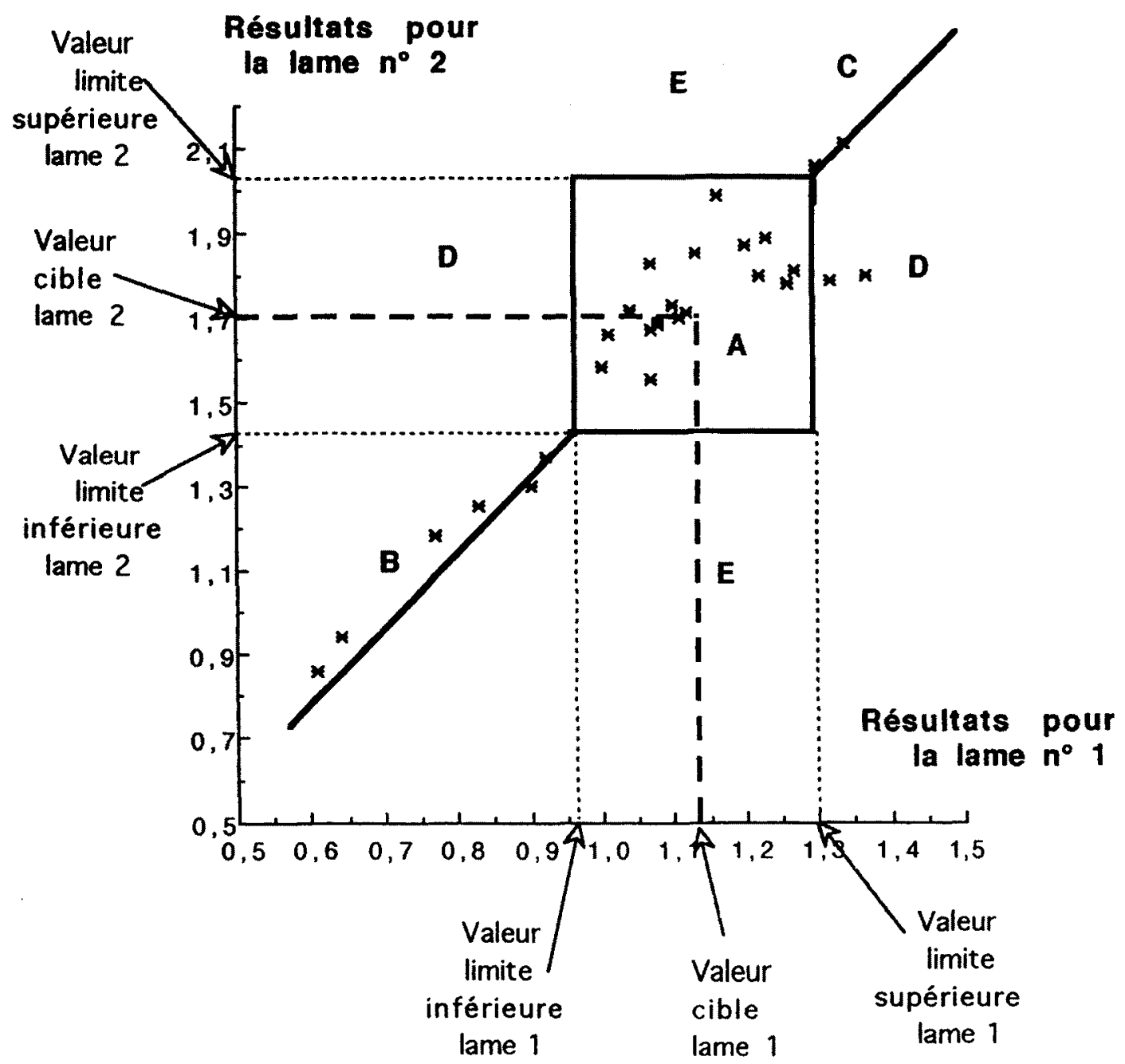

Figure 2 : Diagramme de Youden permettant d'interpréter les résultats d'une analyse de laboratoire en termes de précision et d'exactitude. 
- Zone "A" : Résultats acceptables en termes de précision et d'exactitude.

- Zone "B" : Résultats situés hors de la zone d'acceptabilité, positionnés le long d'une droite dite de "précision mais d'inexactitude" car présentant une erreur systématique par défaut.

- Zone "C" : Même raisonnement que pour $B$, mais avec une erreur systématique par excès.

- Zones “D, E" : Imprécisions pour les lames $n^{\circ} 1$ et 2 respectivement (un des deux résultats se trouve dans la zone d'acceptabilité, l'autre pas).

Pour apprécier le degré d'accord entre les résultats de chaque participant et la valeur à atteindre, cette dernière se doit d'être déterminée de la meilleure façon possible. Compte-tenu du petit nombre de participants au début, nous avons retenu dans un premier temps la notion de valeur-cible calculée de la manière suivante : elle est issue de 10 lectures sur la même lame, à la même place, par le même observateur, considéré comme expérimenté, à des temps différents ; puis 5 lectures sur 5 lames différentes par le même observateur. A côté de la valeur "cible", il nous a aussi fallu définir le degré de variabilité acceptable des résultats pour un même paramètre (appelé aussi limite d'erreur tolérable, ou zone d'acceptabilité des résultats). Ce paramètre prend en compte la qualité analytique de mesure à un moment donné et peut ainsi être réactualisé compte tenu des progrès analytiques enregistrés pour le dit paramètre. Nous avons arbitrairement choisi le seuil de $15 \%$ pour les deux paramètres sachant que cette valeur peut être réévaluée si nécessaire.

\section{RESULTATS}

\section{Nombre de participants}

D'avril 1992 à décembre 1996, le nombre de participants a été multiplié par 2,5, passant de 18 à 47, ce qui montre bien l'intérêt sus- cité par cette démarche, nouvelle dans ce domaine.

\section{Etude des formes normales}

Après étude des valeurs du premier CQE d'avril 1992 (Figure 3-A), on note une très grande dispersion des 18 résultats et qu'un laboratoire seulement (soit $6 \%$ ) se trouve dans la zone d'acceptabilité ; en novembre 1996 (Figure 3-B), les 47 points représentant les laboratoires participants sont plus regroupés et parmi eux, 11 (23\% des participants) se trouvent dans la zone d'acceptabilité.
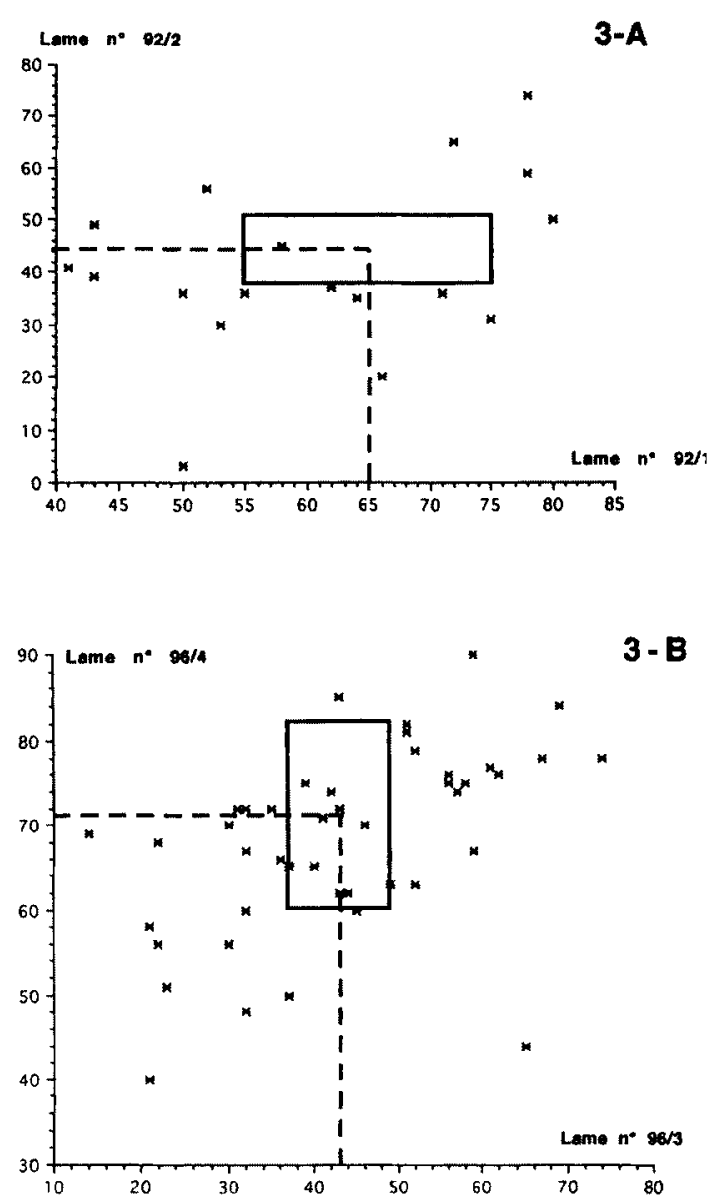

Figure 3 : Evaluation de la proportion de spermatozoïdes normaux (pour 100 éléments comptés) en avril 1992 (3A) et en novembre 1996 (3B).

. . . . . . . : Valeurs cibles $\pm 15 \%$. 


\section{Etude des formes anormales}

L'étude des formes anormales s'est faite par l'utilisation de l'IAM.

En avril 1992, on note une très nette sousestimation du degré de tératospermie (Figure 4-A) et un seul participant (6\%) se trouve dans la zone d'acceptabilité; en novembre 1996, le regroupement des points le long de la droite d'inexactitude indique une très nette amélioration de la précision des résultats. De plus, la cohérence est meilleure puisque 20 points se trouvent dans la zone d'acceptabilité (soit $43 \%$ des participants) (Figure 4-B).
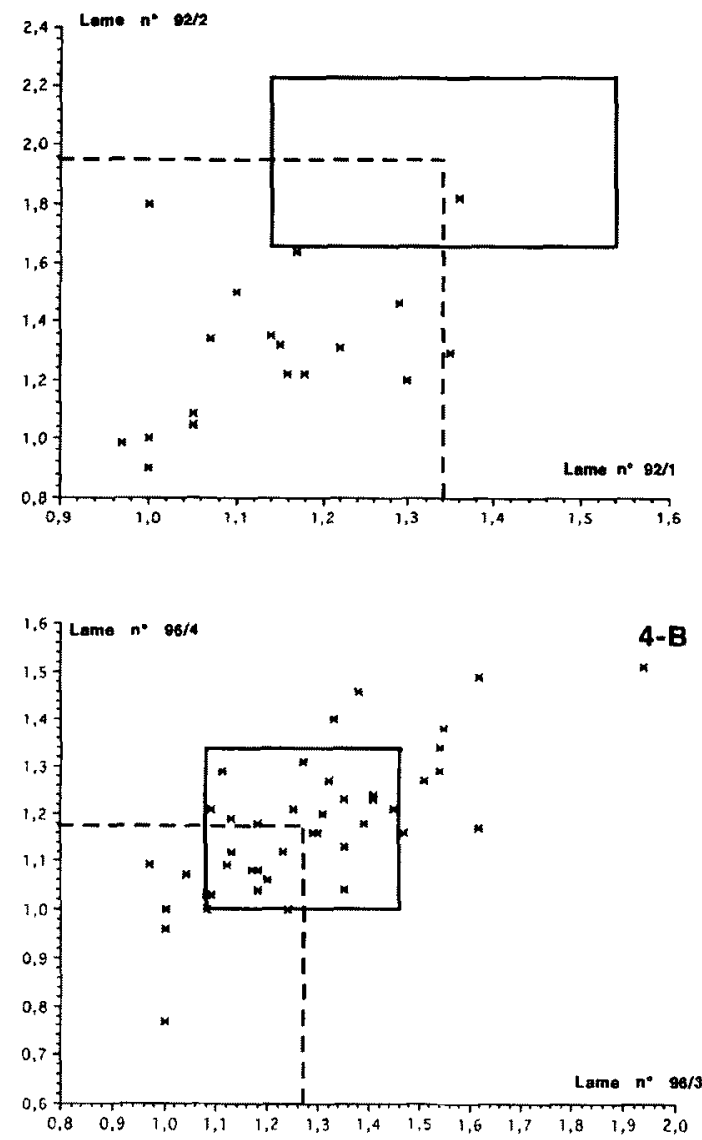

Figure 4 : Evaluation de l'LAM en avril 1992 (4A) et novembre 1996 (4B).

. . . . . . . . . Valeurs cibles $\pm 15 \%$.

\section{Evolution de la dispersion des résul-} tats

L'étude de ce paramètre se fait à l'aide du coefficient de variation (CV). Concernant la proportion des formes normales, la valeur des CV est toujours importante (de 20 à 50 $\%$ ), ce qui montre bien la difficulté actuelle à standardiser cette mesure et l'effort qui reste à faire dans ce domaine (Figure 5). Par contre, le niveau de dispersion des valeurs des CV de l'IAM est très encourageant puisqu'il se situe dans la zone des 15 $\%$, et ce depuis pratiquement le début (Figure 6).

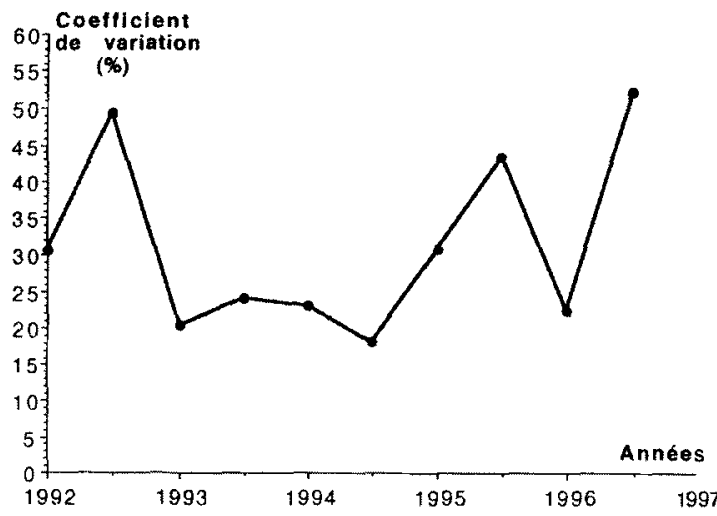

Figure 5 : Evolution de la dispersion interlaboratoire des résultats de l'évaluation de la proportion de spermatozoïdes normaux de 1992 à 1996.

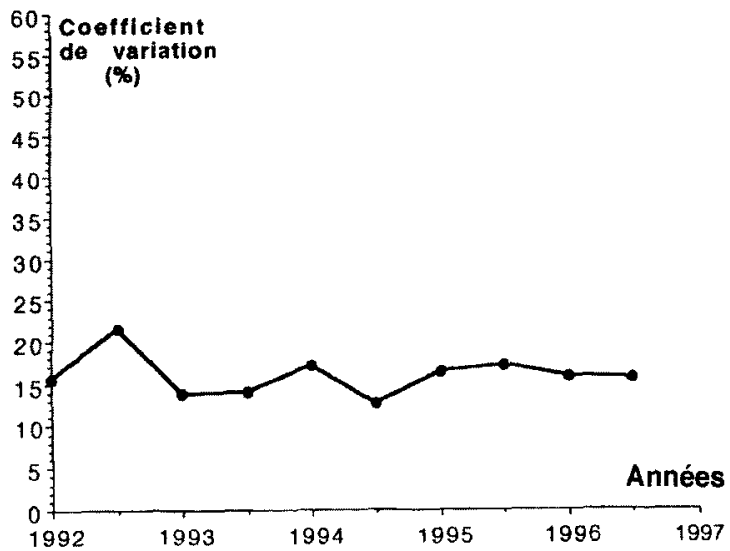

Figure 6 : Evolution de la dispersion interlaboratoire des résultats de l'évaluation de l'IAM de 1992 à 1996. 
La notion d'assurance de qualité en Biologie de la Reproduction a pris une ampleur certaine puisqu'un nombre de plus en plus important de laboratoires pratiquant des analyses dans ce domaine se mobilise pour augmenter la cohérence interlaboratoire des résultats. Par ailleurs, peu d'expériences de CQE ont été décrites jusqu'à maintenant. Celle que nous rapportons ici montre la transférabilité des méthodes utilisées dans les CQE de Biochimie Clinique vers des domaines qui relèvent plus de l'observation que de la mesure comme le spermocytogramme. La mise en place d'une telle démarche a permis de réduire les dispersions importantes de résultats en augmentant la précision des mesures dans certains cas, ou à mettre en évidence des difficultés de standardisation. La seconde étape consiste à aborder la notion d'exactitude, notion beaucoup plus complexe qui va faire l'objet des prochaines réunions du groupe de travail "Assurance de Qualité en Biologie de la Reproduction". De plus, si le nombre d'adhérents continue de progresser, nous comparerons aussi la qualité des résultats avec les différentes techniques de réalisation du spermocytogramme (techniques de coloration, grossissement utilisé, ...) afin d'affiner la valeur diagnostique ou pronostique des résultats produits par le laboratoire.

\section{REFERENCES}

1. David G., Bisson J.-P., Czyglik F., Jouannet P., Gernigon C. : Anomalies morphologiques du spermatozoide humain. I . Proposition pour un système de classification. J. Gynécol. Obstr. Biol. Reprod., $1975,4: 17-36$.

2. Jouannet P., Ducot B., Feneux D. : Male factors and the likelihood of pregnancy in infertiles couples. I. Study of sperm characteristics. Int. J. Androl. 1988, 11 : 379-394.

3. Youden W.J. : Statistical techniques for Collaborative Tests. Assoc. of Official Analytical Chemist, Washington, D.C., 1967.
External Quality Control in spermiology from 1992 to 1996

Bourguignat A., Clavert A., Férard G. Clinical efficiency of laboratory results in spermiology needs to have reliable data, and a quality assurance in their determinations. This could be done by creating an External Quality Control, each laboratory analyzing the same specimens. We transferred in spermiology methodologies currently used in Quality Control in Biochemistry and results presented here referred to the $1992-1996$ period.

Volountary participation of 37 laboratories who recieved two slides issuing from the same ejaculate. Coloration was made with that used in their own laboratory. Two parameters were specially studied : the total normal sperm count and the degree of teratospermia by evaluation of the Multiple Abnormalities Index (MAI). An area around the target value of $15 \%$ is actually retained and results in terms of precision and accuracy are interpreted with the Youden's diagram.

For the total normal sperm count, the disperson remains important during the 4 years (about 30 and $40 \%$ ) with a poor percentage of laboratories in the area of accuracy. For the MAI, results are better as soon as the dispersion falls from 25 to $15 \%$ during the same time and the percentage of laboratories in the accuracy area rises from $10 \%$ to $68 \%$.

After four years of external quality control, such a protocol is very useful. But the quality results of some parameters must be more extensively explored to reduce interlaboratory dispersion and to improve the clinical efficiency of laboratory data in the spermiology approach.

Key-words : External Quality Control - Spermiology. 